\title{
Avaliação das limitações do comportamento social dos moradores dos serviços residências terapêuticos de um pequeno município do estado do Rio de Janeiro
}

\author{
Assessment of social disablement among individuals \\ living in therapeutic residential services in a small municipality \\ of Rio de Janeiro state
}

\author{
Dulce Anny Alves Medeiros¹, Lúcia Abelha, Diego de Lima Fonseca¹, \\ Keli Sarução ${ }^{1}$, Giovanni Marcos Lovisi ${ }^{1}$
}

\begin{abstract}
Resumo
Introdução: Conhecer a dimensão das limitações do comportamento social em pessoas com transtornos mentais graves habitantes de serviços residenciais terapêuticos (SRT) é crucial para gestores e profissionais de saúde mental envolvidos na prestação de cuidados baseados na comunidade. Assim, realizou-se um estudo transversal em indivíduos que residem nesses serviços em uma pequena cidade do Estado do Rio de Janeiro (Carmo). Objetivo: Avaliar limitações do comportamento social dos entrevistados através da Escala de Comportamento Social (SBS). Método: Os dados foram coletados em Janeiro/Fevereiro, 2017, utilizando a SBS. Resultados: A amostra foi composta majoritariamente de homens, com idade média de 60,4 anos, analfabetos, com esquizofrenia e outros transtornos psicóticos, provenientes do Estado do Rio de Janeiro, sem contato com suas famílias, com um longo período de hospitalização prévia e recebendo benefícios. Aproximadamente $96 \%$ dos residentes mostraram, pelo menos, um problema de comportamento social, frequentemente relacionados à aparência pessoal/higiene, comunicação: tomando a iniciativa (a pessoas inicia as conversações?), lentidão, rir e falar sozinho, comportamento não especificado (qualquer outro comportamento ou atitude não previamente especificado, que parece estar atrasando o progresso da pessoa). Conclusão: $\mathrm{A}$ população estudada apresentou alta frequência de limitação de problema social, podendo impedir sua reintegração, tornando-se necessário implementar programas de reabilitação social para este grupo.
\end{abstract}

Palavras-chave: limitações do comportamento social; serviços residenciais terapêuticos; transtornos mentais graves.

\begin{abstract}
Background: Knowledge about the extent of social disablement among people with severe mental disorders living in therapeutic residential services is crucial for policy makers and mental health professionals involved in the delivery of community-base care. A cross-sectional study was conducted with people living in therapeutic residential services in the municipality of Carmo, Rio de Janeiro state, Brazil. Objective: Evaluate limitations of the social behavior of the interviewees through the Social Behavior Scale (SBS). Method: Data were collected between January and February 2017 using the SBS. Results: The sample was composed mostly of men from Rio de Janeiro state, with mean age of 60.4 years, illiterate, benefit recipients, with schizophrenia and other psychotic disorders and a long period of previous hospitalization with no contact with their families. Approximately $96 \%$ of them

1'nstituto de Estudos em Saúde Coletiva (IESC), Universidade Federal do Rio de Janeiro (UFRJ) - Rio de Janeiro (RJ), Brasil.

Trabalho realizado no Instituto de Estudos em Saúde Coletiva (IESC), Universidade Federal do Rio de Janeiro (UFRJ) - Rio de Janeiro (RJ), Brasil.

Endereço para correspondência: Dulce Anny Alves Medeiros - Instituto de Estudos em Saúde Coletiva (IESC), Universidade Federal do Rio de Janeiro (UFRJ), Avenida Horácio Macedo, s/n, Cidade Universitária - Ilha do Fundão - CEP: 21941-598 - Rio de Janeiro (RJ), Brasil - Email: dulceanny_ri@yahoo.com.br

Fonte de financiamento: nenhuma.

Conflito de interesses: nada a declarar.
\end{abstract}

Este é um artigo publicado em acesso aberto (Open Access) sob a licença Creative Commons Attribution, que permite uso, distribuição e reprodução em qualquer meio, sem restrições desde que o trabalho original seja corretamente citado. 
showed at least one social behavior problem, often related to poor self-care, little spontaneous communication (Does the person start a conversation?), slowness, laughing and talking to self, and other problems (any other behavior or attitude not previously specified that seem to be delaying the progress of the person). Conclusion: This population presented high frequency of social disablement that may prevent their social reintegration, evidencing the need for implementing social rehabilitation programs.

Keywords: social disablement; therapeutic residential services; severe mental disorders.

\section{INTRODUÇÃO}

Os Serviços Residenciais Terapêuticos (SRTs) surgiram como uma proposta de moradia para as pessoas portadoras de transtornos mentais graves, que estiveram por longos anos internadas em instituições psiquiátricas e para os egressos de hospitais de custódia que estavam desconectados dos familiares e sem apoio social. Essas moradias também podem fornecer apoio a usuários de outros serviços de saúde mental, que não estiveram asilados, porém sem vínculo familiar e social capazes de fornecer-lhes um morar apropriado. O objetivo dessas moradias é possibilitar a acolhida dessas pessoas na comunidade, o retorno a vida na comunidade, incentivar o resgate de sua autonomia e das práticas do cotidiano, ou seja, promover a progressiva inclusão social do morador de forma articulada com a rede de atenção psicossocial ${ }^{1-3}$.

Entretanto, promover a mudança da assistência psiquiátrica, a reinserção social dos indivíduos que viveram durante anos de sua vida asilados, bem como a construção de uma rede territorial estruturada ainda é um desafio para as políticas de Saúde $\mathrm{Mental}^{4}$. São pessoas afastadas há tempos da sociedade e da família, submetidas aos efeitos deletérios da institucionalização prolongada, com vínculos sociais esmaecidos e limitações do comportamento social ${ }^{5-8}$.

Os indivíduos com transtornos mentais graves com longa permanência em instituições psiquiátricas podem apresentar limitações no comportamento social que os prejudicam nas suas reinserções na comunidade. Estas limitações resultam muito mais das dificuldades apresentadas pelo paciente no convívio social do que da sintomatologia psiquiátrica apresentada ${ }^{9}$.

Enquanto o diagnóstico psiquiátrico não é um preditor do prognóstico de reabilitação de um paciente, o comportamento social tem-se mostrado importante na promoção do planejamento das ações necessárias à reinserção dos pacientes na comunidade, sendo mais preditivo do que os sintomas psiquiátricos para a avaliação da reabilitação social de pacientes ${ }^{10,11}$.

As pessoas com transtornos mentais graves são consideradas como pacientes crônicos que padecem de uma doença que se prolonga ao longo do tempo e que afeta a sua qualidade de vida, limitando suas capacidades e tornando-os dependente de um atendimento de saúde mental contínuo. Embora seja complexo mensurar a duração e a severidade da limitação do comportamento social em indivíduos com transtornos mentais graves, algumas escalas já vêm sendo utilizadas para avaliar as limitações do comportamento social de pacientes psiquiátricos ${ }^{9,12,13}$. Dentre essas escalas, destaca-se a Social Behaviour Schedule (SBS).
Wykes e Sturt ${ }^{11}$ realizaram um estudo com o objetivo de avaliar a confiabilidade e a validade da Social Behaviour Schedule em 66 pacientes com longo período de internação. Os autores demonstraram uma alta confiabilidade e validade da SBS, o que a torna recomendada para avaliação de problemas sociais em pacientes que necessitarão de serviços psiquiátricos por um longo período. Em Londres, foi conduzido o projeto Team for the Assessment of Psychiatric Services (TAPS) que avaliou, dentre outros fatores, as limitações do comportamento social de 670 pacientes desospitalizados utilizando a Social Behaviour Schedule (SBS). Esse projeto foi criado em 1985 com o objetivo de avaliar a política nacional de transferência de pacientes de longo período de internação para serviços de saúde mental comunitários na Inglaterra ${ }^{14}$.

No Brasil, são escassos os estudos que avaliaram as limitações do comportamento social em indivíduos com transtornos mentais graves, principalmente aqueles moradores de residências terapêuticas. Abelha et al. ${ }^{15}$ realizaram um estudo seccional em uma população de 881 pacientes psiquiátricos de longa permanência no Instituto Municipal Juliano Moreira/Rio de Janeiro, utilizando três instrumentos, dentre esses: Avaliação das Limitações no Comportamento Social (SBS-BR) e o Inventário de Habilidades de Vida Independente para pacientes psiquiátricos - (ILSS-BR). Os pesquisadores encontraram altas taxas de problemas nas limitações do comportamento social e pouca autonomia em $75 \%$ da população estudada, sugerindo a implementação de um programa de reabilitação social específico para esses indivíduos.

Um estudo de coorte não comparada foi realizado em uma população de 75 pacientes com longo período de internação. Esses pacientes foram avaliados em 2 momentos: antes da transferência do hospital e a após 2 anos vivendo na comunidade. Os autores avaliaram além do comportamento social, a autonomia desses indivíduos. Foi evidenciada uma melhora significativa no comportamento social desses indivíduos na segunda etapa da pesquisa, apesar de ainda permanecerem limitações quanto à aparência, comunicação e interação social ${ }^{16}$.

Em um estudo transversal realizado na região Sul do país as limitações do comportamento social forma avaliadas com o uso da SBS em 385 indivíduos usuários de dois tipos de serviços substitutivos: Serviço Residencial Terapêutico e CAPs. Os pesquisadores encontraram uma maior prevalência de comportamentos inespecíficos em ambos os serviços, seguido de ataques de pânico em usuários de CAPS e problemas com aparência pessoal e de higiene em usuários de SRTs ${ }^{17}$. 
A aferição das limitações do comportamento social tem função primordial no planejamento, na avaliação do desempenho dos indivíduos com transtornos mentais graves na comunidade e na estruturação de programas de reabilitação social que contemplem a carência de cuidados psicossociais e físicos a que foram expostos por longo período. Portanto, o objetivo principal do estudo foi o de avaliar as limitações do comportamento social de moradores em residências terapêuticas de um município de pequeno porte do estado do Rio de Janeiro, oriundos de um longo período de internação em hospital psiquiátrico.

\section{MÉTODOS}

\section{Local do estudo}

O estudo foi realizado na cidade de Carmo, região serrana do Rio de Janeiro. O município tem uma área total de 320,7 quilômetros quadrados, correspondentes a 4,6\% da área da Região Serrana. Os limites municipais, no sentido horário, são: Minas Gerais, Cantagalo, Duas Barras, Sumidouro e Sapucaia.

Nesse município foi fundado em 1947, o Hospital Estadual Teixeira Brandão - HETB que se localizava numa fazenda com área de cerca de 160 alqueires, em uma área tipicamente rural. Possuía 400 leitos psiquiátricos exclusivamente para o sexo masculino. No ano de 2000, o hospital sofreu intervenção da Secretária Estadual de Saúde do Rio de Janeiro por más condições na assistência de seus internos.

A assistência psiquiátrica no Município do Carmo é composta por um Centro de Atenção Psicossocial do tipo II, um Centro de Convivência, leitos psiquiátricos no hospital geral do município e Serviços Residenciais Terapêuticos. A cidade possui atualmente 21 Residências Terapêuticas situadas na zona urbana, abrigando um total de 121 moradores, os quais são oriundos do Hospital Estadual Teixeira Brandão. No período de fechamento do hospital foram implantadas 25 Residências Terapêuticas, esse número foi reduzido em decorrência de óbitos e pelo fato de alguns indivíduos terem optado por viverem sozinhos ou com algum familiar em locais escolhidos por eles mesmos.

\section{População do estudo}

A população foi de 121 moradores das residências terapêuticas de Carmo, sendo as informações fornecidas pelos informantes-chaves, constituídos pelos profissionais de saúde que trabalhavam nas residências terapêuticas. Foram excluídos aqueles indivíduos que não aceitarem participar do estudo e aqueles que apresentarem incapacidade de responder a entrevista.

\section{Instrumentos utilizados}

\section{Características sociodemográficas}

Segundo outros estudos desenvolvidos sobre o tema ${ }^{16,17}$, as características sociodemográficas incluíram as seguintes variáveis: sexo, faixa etária, estado civil, local de nascimento, escolaridade, número de filhos, contato com algum parente, tempo de internação anterior em hospital psiquiátrico, doença física, hábito de fumar e benefícios recebidos. O diagnóstico psiquiátrico foi coletado nos prontuários dos moradores, o qual é baseado na Classificação Internacional de Doenças, 10a edição (CID-10).

\section{Escala de Avaliação de Limitações no Comportamento Social (SBS-Br Social Behaviour Schedule - SBS)}

O presente estudo utilizou a SBS, que avalia as limitações do comportamento social de pacientes de longa permanência, tanto em hospitais psiquiátricos quanto em serviços residenciais na comunidade. A escala é composta de 21 itens, a maioria pontuados de 0 a 4 . O último item (item 21) permite ao informante-chave incluir problemas diversos, como alcoolismo e distúrbios alimentares, mas não foram frequentes o suficiente em estudos anteriores para justificá-los como outros itens separadamente na escala ${ }^{11}$.

A escala apresenta duas formas de avaliação. O método BSS (Severe Behaviour Problems Scores), cujo problema é ausente quando a pontuação é 0 , leve quando a pontuação é 1 ou 2 e grave quando a pontuação é 3 ou 4 . O presente estudo utilizou o método de pontuação BSM (Mild and Severe Behaviour Problems Scores). Nesse método o problema é considerado presente quando a pontuação das respostas é 2,3 ou 4 e ausente quando essa pontuação é 0 ou 1 . Wykes e Sturt ${ }^{11}$ agruparam os 21 itens da SBS em 5 componentes, apresentados a seguir:

- Afastamento social: 1, 18, 19;

- Comportamento social embaraçoso: 2, 3,4, 9, 11, 16, 20;

- Depressão e ansiedade: 6, 7, 8, 15;

- Hostilidade e violência: 5,14;

- Comportamento social inaceitável: 12, 13,17, 21.

As informações foram obtidas a partir das observações de um informante-chave que conhecia bem sobre o comportamento do paciente no último mês anterior a pesquisa. A severidade do comportamento e a frequência da ocorrência devem ser levadas em consideração ao ser feita a pontuação. A freqüência da ocorrência deve sempre prevalecer sobre a gravidade do comportamento. Esta escala foi validada para uso no Brasil ${ }^{12}$.

\section{Coleta de dados}

A pesquisadora principal do projeto foi treinada previamente nos dois instrumentos de avaliação. A coleta dos dados foi efetuada em local privado, situado no interior da Secretaria de Saúde de Carmo, garantindo ao participante a privacidade às suas respostas. Os informantes-chaves foram explicados acerca do estudo e após aceitarem participar e assinarem o termo de consentimento, foram perguntados acerca das limitações do 
comportamento social dos moradores das residências terapêuticas utilizando a Social Behaviour Scale (SBS-BR) durante o período de Janeiro a Fevereiro de 2017.

\section{Análise de dados}

Para a análise univariada foram utilizadas as técnicas estatísticas descritivas tais como: frequência, média, e desvio padrão. Os dados coletados foram inseridos em um banco de dados através de uma máscara construída no programa Epiinfo 7. As análises estatísticas foram realizadas utilizando o programa Statistical Package for Social Sciences - SPSS, versão 17.

\section{Aspectos éticos}

O estudo foi autorizado pelo Comitê de Ética em Pesquisa do Instituto de Estudos em Saúde Coletiva da UFRJ (número do parecer de aprovação do comitê de ética: 1.867.892). Antes da entrevista cada informante-chave foi informado sobre a pesquisa e esclarecido sobre suas dúvidas, sendo convidado a participar e assinar um Termo de Consentimento Livre e Esclarecido. Eles foram assegurados de que sua participação seria voluntária, sem prejuízos, tendo plena liberdade para sair do estudo a qualquer momento. Todos os dados foram mantidos em sigilo e os casos identificados através de um código numérico.

\section{RESULTADOS}

A taxa de participação do estudo foi de 86,8\% (n=105). A grande maioria da população estudada é composta por homens, média de 60,4 anos e desvio-padrão de 11,9 de anos, analfabetos, com o diagnóstico de esquizofrenia e outros transtornos psicóticos, oriundos do estado do Rio de Janeiro, sem relacionamento íntimo, sem filhos, sem contatos familiares, com longo período de internação em hospitais psiquiátricos (mais de 20 anos, com doenças físicas (predominando hipertensão arterial e diabetes), não fumantes e recebendo benefícios (Tabela 1). As despesas individuais dos moradores das SRT são providas por fontes como o Programa de Volta pra Casa, LOAS, aposentadoria do INSS, auxílio dos familiares e/ou pensão.

Cerca de 96\% dos moradores apresentaram pelo menos 1 problema de limitação de comportamento social, sendo que $59,4 \%$ apresentaram entre 3 a 6 problemas. Os itens das limitações do comportamento social mais frequentes foram: aparência pessoal e higiene (considera o asseio, cabelos, se troca as roupas de baixo), comunicação: tomando a iniciativa (se a pessoa inicia as conversações), lentidão (considera a lentidão anormal, como se a pessoa anda anormalmente devagar), rir e falar sozinho (evidencia de que a pessoa não está rindo socialmente), inquietação e hiperatividade (como movimentos desnecessários e frequentes, inquietação geral, excitação), conversação: incoerência (dificuldade em manter o dialogo com outras pessoas devido a incoerência do discurso), inatividade
Tabela 1. Distribuição das características sócio-demográficas dos moradores de residências terapêuticas do município de Carmo, RJ, 2016

$$
\text { Características }
$$

$\mathrm{N}=105$ Frequência \%

\section{SEXO}

Masculino

$100 \quad 95,2$

Feminino

5

4,8

FAIXA ETÁRIA (anos)

$25-55$

$56-67$

68-89

33

31,4

SCOLARIDADE

\section{Analfabeto}

Ensino Fundamental incompleto

Ensino médio incompleto

Ensino médio completo

$46 \quad 43,8$

LOCAL DE NASCIMENTO (estados)

Rio de Janeiro

Minas Gerais

Outros*

FILHOS

Sim
Não
COMPANHEIRO (a)

Sim

Não

82

78,1

20

19,0

2

BENEFÍCIOS $* *$

Sim

Não

\section{1}

84,8

89

FUMANTE

Sim

Não

CONTATO FAMILIAR

$\operatorname{Sim}^{\star * *}$

Não

TEMPO DE INTERNAÇÃO (anos)

5-20

21-35

36-50

Não

Sem informação

DOENÇAS FÍSICAS

Sim

Não

DIAGNÓSTICO PSIQUIÁTRICO****

Transtorno Mental Orgânico

Esquizofrenia/Transtornos

Psicóticos

62,9

Retardo Mental

Outros

Sem informação

${ }^{*}$ ão Paulo $=2$; Pernambuco=2; Espírito Santo $=1 ;$ Ceará $=1 ;{ }^{*}$ Programa de

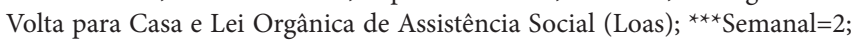
Quinzenal=2; Mensal=6; Bimestral=1; Trimestral=5; Anual=17; Diagnóstico psiquiátrico; ${ }^{* * *}$ de toda a população $(\mathrm{n}=121)$ 
(falta de atividade expontânea), conversação: excentricidade (o quanto a conversação da pessoa mostra uma preocupação com tópicos bizarros ou excêntricos) e comportamento não especificado (qualquer outro comportamento ou atitude como fumar e comer em excesso, anorexia, roubo, obsessões, distúrbio de sono).

Por outro lado, as frequências foram muito baixas nos seguintes itens: hábitos ou maneiras socialmente inaceitáveis, ideais ou comportamento suicida ou de auto-agressão e comportamento destrutivo (Tabela 2). As limitações do comportamento social mais frequentes nos componentes da SBS foram: comportamento social inaceitável e afastamento social (Tabela 3).

Tabela 2. Distribuição das limitações do comportamento social nos moradores de residências terapêuticas do município de Carmo, Rio de Janeiro, 2016

\begin{tabular}{|c|c|c|}
\hline Tipo de Problema & $\mathrm{N}=105$ & Frequência \% \\
\hline Comunicação: Tomando a iniciativa & 41 & 39,0 \\
\hline Conversação: Incoerência & 30 & 28,6 \\
\hline $\begin{array}{l}\text { Conversação: Excentricidade/ } \\
\text { inadequação }\end{array}$ & 28 & 26,7 \\
\hline $\begin{array}{l}\text { Interação Social/Habilidade de fazer } \\
\text { contatos de forma adequada }\end{array}$ & 9 & 8,6 \\
\hline $\begin{array}{l}\text { Interação social: Proporção de contatos } \\
\text { sociais que são por natureza hostil }\end{array}$ & 12 & 11,4 \\
\hline $\begin{array}{l}\text { Interação social: Comportamento } \\
\text { chamativo }\end{array}$ & 25 & 23,8 \\
\hline $\begin{array}{l}\text { Idéias ou comportamento suicida ou de } \\
\text { auto-agressão }\end{array}$ & 1 & 1,0 \\
\hline Ataques de pânico e fobias & 18 & 17,1 \\
\hline Inquietação e hiperatividade & 35 & 33,3 \\
\hline Rir e falar sozinho & 39 & 37,1 \\
\hline Realização de ideias bizarras & 4 & 3,8 \\
\hline Estereotipias e maneirismos & 26 & 24,8 \\
\hline $\begin{array}{l}\text { Hábitos ou maneiras socialmente } \\
\text { inaceitáveis }\end{array}$ & 1 & 1,0 \\
\hline Comportamento destrutivo & 3 & 2,9 \\
\hline Depressão & 5 & 4,8 \\
\hline Comportamento sexual inadequado & 6 & 5,7 \\
\hline Aparência pessoal e higiene & 44 & 41,9 \\
\hline Lentidão & 39 & 37,1 \\
\hline Inatividade & 30 & 28,6 \\
\hline Concentração & 19 & 18,1 \\
\hline $\begin{array}{l}\text { Comportamento não especificado em } \\
\text { outro lugar e que impeça progressos }\end{array}$ & 41 & 39,0 \\
\hline
\end{tabular}

Tabela 3. Distribuição dos componentes das limitações do comportamento social entre os moradores de residências terapêuticas do município de Carmo, Rio de Janeiro, 2016

\begin{tabular}{lcc}
\multicolumn{1}{c}{ Componentes } & N=105 & Frequência \% \\
Afastamento social & 20 & 19,0 \\
Comportamento social embaraçoso & 7 & 6,7 \\
Depressão e ansiedade & 5 & 4,8 \\
Hostilidade e violência & 7 & 6,7 \\
Comportamento social inaceitável & 30 & 28,6 \\
\hline
\end{tabular}

\section{DISCUSSÃO}

O principal resultado do presente estudo demonstrou que a população avaliada apresentou altas frequências de limitações do comportamento social, particularmente nas áreas de comportamento social inaceitável e afastamento social. Praticamente todos os participantes apresentaram ao menos 1 item de limitação do comportamento social e a maior concentração entre 3 a 6 problemas (59,4\%). Esse achado foi similar ao encontrado por Lima et al. ${ }^{18}$, que avaliaram 881 pacientes de um hospital psiquiátrico. Os itens mais frequentes foram aparência pessoal e higiene, comunicação: tomando a iniciativa, comportamento não especificado, lentidão, rir e falar sozinho, inquietação e hiperatividade, conversação: incoerência, inatividade e conversação: excentricidade, os quais variaram de $26,7 \%$ a 41,9\%. Esses mesmos itens foram encontrados com maior frequência em outros estudos nacionais que avaliaram as limitações do comportamento social utilizando a SBS. O presente estudo encontrou uma prevalência muito maior desses itens em comparação aos estudos de Lovisi et al. ${ }^{19}$ e Jardim et al. ${ }^{17}$, que avaliaram população de rua e pessoas em tratamento nos CAPs e residências terapêuticas, respectivamente.

Esse resultado pode ser explicado pela maior vulnerabilidade dos moradores de Carmo como faixa etária mais idosa, maior frequência de transtornos mentais graves, maior período de internação psiquiátrica prévia e sem contato familiar. Por outro lado, as freqüências encontradas no presente estudo foram menores em comparação aos estudos conduzidos por Fleck et al. ${ }^{20}$ e Lima et al. ${ }^{18}$, que realizaram o trabalho em indivíduos internados por um longo período em um hospital psiquiátrico no Rio Grande do Sul e Rio de Janeiro, respectivamente.

$\mathrm{O}$ fato de o indivíduo estar internado por um longo período em um hospital psiquiátrico justificaria uma maior frequência de limitações do comportamento social em comparação à indivíduos que já estão vivendo em comunidades. Esse achado é corroborado pelo estudo conduzido por Vidal et al. ${ }^{7}$, que avaliaram as limitações do comportamento social utilizando a SBS em pacientes psiquiátricos antes da saída do hospital e 2 anos após a transferência para residências terapêuticas. Segundo os autores, os pacientes apresentaram uma evolução satisfatória no comportamento social após 2 anos vivendo nas residências terapêuticas. A razão de viver na comunidade pode propiciar um ambiente mais acolhedor para o indivíduo do que o longo período de internação hospitalar ${ }^{21}$.

Chama a atenção que o comportamento destrutivo e as ideais suicidas foram os menores percentuais de toda a lista de 21 itens da SBS em todos os estudos nacionais. Esse fato pode sugerir que indivíduos que vieram de longo período de internação, e com maior comprometimento nas suas limitações, podem apresentar baixo risco a si mesmo e aos demais ${ }^{17}$. 
Wykes e Sturt ${ }^{11}$ conduziram um estudo em Londres com o objetivo de avaliar as limitações do comportamento social em pacientes psiquiátricos com longo período de internação $(\mathrm{n}=66)$ atendidos em serviços de saúde mental na comunidade ou internados em um hospital psiquiátrico. Comparando com os resultados do presente estudo, os moradores de Carmo mostraram uma maior frequência de problemas em quase todos os itens da SBS, exceto interação social, hostilidade e ataques de pânico. Uma das explicações para esse fato pode ser os serviços de reabilitação psicossocial oferecidos aos pacientes de Londres.

Um outro estudo realizado em Londres com o objetivo de avaliar os efeitos associados com a transferência de pacientes idosos com longo período de internação psiquiátrica $(n=165)$ para serviços de saúde mental na comunidade. Vários instrumentos foram utilizados, incluindo o SBS. Após 1 ano do início do estudo os autores não encontraram mudanças significativas nos problemas do comportamento social dos participantes ${ }^{22}$. O mesmo resultado foi encontrado no estudo do projeto Team for the Assessment of Psychiatric Services (TAPS), que avaliou após 1 ano do início do estudo, 670 pacientes desospitalizados utilizando a $\mathrm{SBS}^{14}$.

Entretanto, outro estudo prospectivo com pacientes de longo período de internação e classificados como inadequados para os serviços de saúde mental comunitários, apresentou melhora significativa do comportamento social somente após 5 anos do início do estudo. Os autores alegaram que os pacientes com logo período de internação necessitam um período maior de reabilitação psicossocial intensiva para apresentar melhoras no comportamento social ${ }^{23}$.

Os resultados das características socio-demográficas demonstram a grande frequência de adversidades sociais que afetam os moradores das residências terapêuticas de Carmo. Apesar de receberem benefícios, a grande maioria dessa população é composta por homens, idosos, analfabetos, com diagnóstico de esquizofrenia e outros transtornos psicóticos, com longo período anterior de internações em hospitais psiquiátricos e sem contato familiar.

O afastamento social é um grave problema psicossocial que ainda afeta essas pessoas. Apesar de já estarem morando em residências na comunidade há mais de 10 anos, esses indivíduos continuam com dificuldades de estabelecerem vínculos familiares. Esse achado está em consonância com o resultado do projeto TAPS ${ }^{14}$ e com o estudo realizado por Thornicroft \& Tansella ${ }^{21}$, os quais demonstraram que a rede social dos pacientes não aumentou significativamente para a grande maioria dos participantes após a desospitalização. Entretanto, houve uma melhora na qualidade de vida desses indivíduos, que preferiram continuar vivendo na comunidade.

Apesar de receber benefícios, a grande maioria dos moradores dos SRTs de Carmo apresenta uma grande proporção de problemas no comportamento social, na vulnerabilidade social e uma rede social restrita, o que poderia ser explicado pelo longo período de internação psiquiátrica prévia e abandono. A alta frequência de problemas sociais dificulta a reinserção social desses moradores em SRTs, podendo levar ao afastamento social.

Levando em conta que a atual crise econômica do Brasil / Rio de Janeiro é grave, o critério de decisão em muitas áreas cruciais, como a saúde, é baseado principalmente na relação custo-benefício ${ }^{24}$. Infelizmente, o principal objetivo dessas decisões é geralmente reduzir custos (e consequentemente os benefícios conferidos aos pacientes), o que, sem dúvida, leva a uma diminuição da qualidade dos cuidados. Isto afeta ainda mais as desigualdades sociais na utilização dos serviços de saúde no Brasil, principalmente nas populações mais vulneráveis, como os indivíduos com transtornos mentais graves com problemas socioeconômicos e sem um apoio social adequado.

A avaliação do comportamento social pela SBS é fundamental para a implementação de programas de reabilitação psicossocial para indivíduos com transtornos mentais graves em tratamento hospitalar ou em serviços comunitários. Leff e Szmidla ${ }^{22}$ avaliaram um programa de reabilitação psicossocial especialmente desenvolvido para pacientes hospitalizados e classificados com dificuldades para serem transferidos para serviços comunitários. Os programas de reabilitação eram individualizados e focados em cada problema social e déficit de habilidades sociais. Os autores concluíram que o programa individualizado e intensivo (incluindo a terapia cognitiva comportamental), o treinamento dos cuidadores nesses programas e o uso adequado da medicação foram cruciais para a melhora do comportamento social de alguns pacientes.

Como se trata de um Estudo Epidemiológico Seccional Descritivo há problemas com relação ao viés temporal, visto que é difícil separar causa e efeito no presente estudo. Outra limitação foi a utilização de uma amostra populacional de conveniência e não probabilística, o que limita a generalização dos resultados.

Os moradores de residências terapêuticas da cidade de Carmo apresentaram uma alta frequência de limitações do comportamento social, sobretudo nas áreas de comportamento social inaceitável e afastamento social. Comparando os resultados do presente estudo com os achados de outros estudos nacionais e internacionais, os problemas sociais encontrados nos moradores dos SRTs de Carmo foram mais frequentes em comparação aos indivíduos em tratamento comunitário e menos frequentes em comparação aos pacientes internados em hospitais psiquiátricos.

As características socio-demográficas demonstram grande frequência de adversidades sociais que afetam os moradores das residências terapêuticas de Carmo. Apesar de receberem benefícios, a grande maioria dessa população é composta por homens, idosos, analfabetos, com diagnóstico de esquizofrenia e outros transtornos psicóticos, com longo período anterior de internações em hospitais psiquiátricos, com doenças físicas e 
sem contato familiar. $\mathrm{O}$ afastamento social é um grave problema psicossocial que ainda afeta essas pessoas.

A aferição das limitações do comportamento social tem função primordial no planejamento, na avaliação do desempenho dos indivíduos com transtornos mentais graves na comunidade e na estruturação de programas de reabilitação psicossocial que contemplem a carência de cuidados psicossociais e físicos a que foram expostos por longo período este grupo populacional.

\section{REFERÊNCIAS}

1. Argiles CTL, Kantorski LP, Willrich JQ, Antonacci MH, Coimbra VCC. Redes de sociabilidade: construções a partir do serviço residencial terapêutico. Ciênc. Saúde Coletiva. 2013;18(7):2049-58. http://dx.doi.org/10.1590/ S1413-81232013000700020

2. Brasil. Ministério da Saúde. Secretaria de Atenção à Saúde. Departamento de Ações Programáticas Estratégicas. Coordenação Nacional de Saúde Mental. Residências terapêuticas: o que são, para que servem? Brasília: Ministério da Saúde; 2004.

3. Brasil. Ministério da Saúde. Portaria $\mathrm{n}^{\circ} 106$ de 11 de fevereiro de 2000. Diário Oficial da União, Brasília, 24 de fevereiro de 2000.

4. Lucena MAS, Bezerra AFB. Reflexões sobre a gestão de processos de desinstitucionalização. Cien Saude Colet. 2012;17(9):2447-56. http://dx.doi. org/10.1590/S1413-81232012000900025.

5. Pande MNR, Amarante PDC. Desafios para os Centros de Atenção Psicossocial como serviços substitutivos: a nova cronicidade em questão. Cien Saude Colet. 2011;16(4):2067-76. http://dx.doi.org/10.1590/S141381232011000400006. PMid:21584448.

6. Gonçalves S, Fagundes P, Lovisi G, Lima LA. Avaliação das limitações no comportamento social em pacientes psiquiátricos de longa permanência. Cien Saude Colet. 2001;6(1):105-13. http://dx.doi.org/10.1590/S141381232001000100009 .

7. Vidal CEL, Bandeira M, Gontijo ED. Reforma psiquiátrica e serviços residenciais terapêuticos. J Bras Psiquiatr. 2008;57(1):70-9. http://dx.doi. org/10.1590/S0047-20852008000100013.

8. Goffman E. Manicômios, prisões e conventos. Tradução Dante Moreira Leite. 9. ed. São Paulo: Perspectiva; 2015.

9. Abelha L, Golçalves S, Lovisi G. Limitações no comportamento social. In: Bandeira M, Abelha L, Barroso S, editores. Avaliação de serviços de saúde mental. Petrópolis: Vozes; 2014.

10. Lovisi GM. Avaliação de distúrbios mentais em moradores de albergues públicos das cidades do Rio de Janeiro e de Niterói [tese]. Rio de Janeiro: Fundação Oswaldo Cruz; 2000.

11. Wykes T, Sturt E. The measurement of social behaviour in psychiatric patients: an assessment of the reliability and validity of the SBS schedule. The BJPsych. 1986;148(1):1-11. PMid:3082403.

12. Lima LA, Gonçalves S, Lovisi G, Pereira BDB. Validação transcultural da Escala de Avaliação de Limitações no comportamento Social - SBS-BR. Arch Clin Psychiatry. 2003;30(4):126-38. http://dx.doi.org/10.1590/S010160832003000400003.

13. Desviat M. A reforma psiquiátrica. Tradução Vera Ribeiro. 2. ed. Rio de Janeiro: Fiocruz; 2008.
14. Leff J, Trieman N. Long-stay patients discharged from psychiatric hospitals. The BJPsych. 2000;176(3):217-23. PMid:10755067.

15. Abelha L, Muñoz MD, Gonçalves S, Fagundes P, Barbosa DR, Legay LF, et al. Evaluation of social disablement, psychiatric symptoms and autonomy in long-stay psychiatric patients. Arch Clin Psychiatry. 2006;33(1):10-7. http://dx.doi.org/10.1590/S0101-60832006000100002.

16. Vidal CEL, Gontijo ECDM, Bandeira MB. Avaliação das habilidades de vida independente e comportamento social de pacientes psiquiátricos desospitalizados. Rev Psiquiatr Rio Gd Sul. 2007;29(3):294-304. http:// dx.doi.org/10.1590/S0101-81082007000300009.

17. Jardim VMR, Kantorski LP, Oliveira MM, Treichel CAS, Rodrigues CGSS, Dias LV. Limitações de comportamento social entre usuários da Rede de Atenção Psicossocial no sul do Brasil. Ciênc. Saúde Coletiva. 2015;20(5):13718. http://dx.doi.org/10.1590/1413-81232015205.15262014.

18. Lima LA, Gonçalves S, Pereira BB, Lovisi GM. The measurement of social disablement and assessment of psychometric properties of the Social Behaviour Schedule (SBS-BR) in 881 Brazilian long-stay psychiatric patients. .' Int J Soc Psychiatry. 2006;52(2):101-9. http://dx.doi.org/10.1177/0020764006062091. PMid:16615243.

19. Lovisi GM, Mann AH, Coutinho E, Morgado AF. Mental illness in an adult sample admitted to public hostels in the Rio de Janeiro metropolitan area, Brazil. Soc Psychiatry Psychiatr Epidemiol. 2003;38(9):493-8. http://dx.doi. org/10.1007/s00127-003-0666-8. PMid:14504732.

20. Fleck MPDA, Wagner L, Wagner M, Dias M. Long-stay patients in a psychiatric hospital in Southern Brazil. Rev Saude Publica. 2007;41(1):12430. http://dx.doi.org/10.1590/S0034-89102007000100017. PMid:17273643.

21. Thornicroft G, Tansella M. Growing recognition of the importance of service user involvement in mental health service planning and evaluation. Epidemiol Psichiatr Soc. 2005;14(1):1-3. http://dx.doi.org/10.1017/ S1121189X00001858. PMid:15792287.

22. Leff J, Szmidla A. Evaluation of a special rehabilitation programme for patients who are difficult to place. Soc Psychiatry Psychiatr Epidemiol. 2002;37(11):532-42. http://dx.doi.org/10.1007/s00127-002-0578-z. PMid:12395143.

23. Trieman N, Leff J. Long-term outcome of long-stay psychiatric in-patients considered unsuitable to live in the community. The BJPsych. 2002;181(5):42832. PMid:12411270.

24. Koyanagi C, Manes J, Surles R, Goldman HH. On being very smart: the mental health community's response in the health care reform debate. Hosp Community Psychiatry. 1993;44(6):537-9. PMid:8514297.

Recebido em: Ago. 01, 2018 Aprovado em: Fev. 27, 2018 